\title{
Emerging Treatment Options for B-Cell Lymphomas
}

Presented by Andrew D. Zelenetz, MD, PhD

\begin{abstract}
As targeted therapy in B-cell lymphomas rapidly expands beyond anti-CD20 monoclonal antibodies, many newer types of agents are in various stages of development. During his presentation at the NCCN 20th Annual Conference, Dr. Andrew D. Zelenetz explored many of them, including the newer immunoconjugates, the Bruton's tyrosine kinase inhibitor ibrutinib, agents targeting apoptosis such as venetoclax (ABT-199), the immunomodulator lenalidomide, and novel immune checkpoint inhibitors such as nivolumab. To improve outcomes in patients with B-cell lymphomas, these therapies either target the tumor cells and their pathways or focus on the microenvironment and immune modulation. (J Natl Compr Canc Netw 2015;13:666-669)
\end{abstract}

A creditable challenge in the development of targeted therapy for B-cell lymphomas (Figure 1) is to develop new clinical trial strategies to streamline the evaluation of novel treatment combinations, declared Andrew D. Zelenetz, MD, PhD, Vice Chair, Medical Informatics, Department of Medicine, Memorial Sloan Kettering Cancer Center; Professor of Medicine at Weill Medical College of Cornell University; and Chair of the NCCN Non-Hodgkin's Lymphomas (NHL) Panel. "We

Presented by Andrew D. Zelenetz, MD, PhD, Vice Chair, Medical Informatics, Department of Medicine, Memorial Sloan Kettering Cancer Center, and Professor of Medicine at Weill Medical College of Cornell University, New York, New York.

Dr. Zelenetz has disclosed that he has received consultant fees/ honoraria from Amgen Inc., Bullet Bio, Cancer Genetics, Inc.,

Celgene Corporation, Dr. Reddy's Laboratories, Emergent

BioSolutions Inc., Foundation Medicine, Inc., Gilead Sciences,

Inc, Hospira, Inc., and sanofi-aventis U.S.; served as a scientific

advisor for Boehringer Ingelheim $\mathrm{GmbH}$, Genentech, Inc., and

Roche Laboratories, Inc.; and received grants or research support

from Abbott Laboratories, Celgene Corporation, Constellation

Pharmaceuticals, Curis, Inc., Genentech, Inc., Gilead Sciences,

Inc, GlaxoSmithKline, Infinity Pharmaceuticals Inc., Jannsen

Pharaceutica Products, LP, Novartis Pharmaceuticals Corporation,

Onyx Pharmaceuticals, Inc., and Seattle Genetics, Inc.

Correspondence: Andrew D. Zelenetz, MD, PhD, Memorial Sloan

Kettering Cancer Center, 1275 York Avenue, Box 330, New York, NY

10065. E-mail: zeleneta@mskcc.org have too many drugs to test and not enough patients to test them in," he admitted. Furthermore, with a host of available and emerging B-cell treatment options, better preclinical models are needed to help screen for the most promising combinations of therapies, and correlative biomarker/somatic mutation analysis is essential to enhance precision in the selection of treatment options in specific subgroups of patients.

\section{Novel Monoclonal Antibodies and Antibody Drug Conjugates}

Joining rituximab as approved anti-CD20 antibodies in the treatment of B-cell lymphomas are ofatumumab and obinutuzumab. Dr. Zelenetz briefly reviewed the supporting data behind these 2 newer agents.

In a randomized phase II study, ${ }^{1}$ the combination of ofatumumab and CHOP (cyclophosphamide, doxorubicin, prednisone, and vincristine) in previously untreated patients with follicular lymphoma (FL) "was clearly active," said Dr. Zelenetz, with $62 \%$ of patients experiencing a complete response. Of note, even many of those in the high-risk FLIPI (Follicular Lymphoma International Prognostic Index) group had a response to treatment. Unfortunately, however, ofatumumab has little to no activity in patients with rituximab-refractory FL, revealed Dr. Zelenetz, with an overall response rate of about $10 \%$.

The phase II GAUSS study ${ }^{2}$ was the first head-tohead study of obinutuzumab versus rituximab in patients with relapsed indolent NHL with a prior response to rituximab. Although a trend toward higher response rates was seen with obinutuzumab, no difference in progression-free survival was noted at this early time point, added Dr. Zelenetz. Phase III studies are comparing obinutuzumab with rituximab in combination 


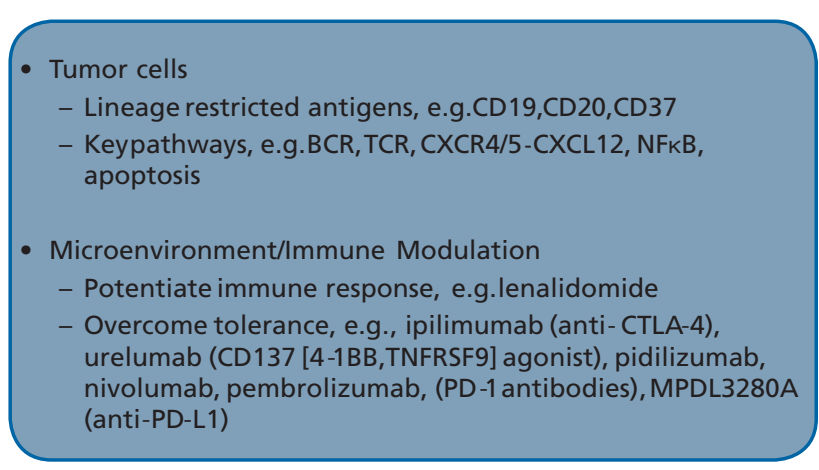

Figure 1 Targeting lymphoma.

with chemotherapy in diffuse large B-cell lymphoma (DLBCL) and FL.

Blinatumomab, a T-cell-engaging bispecific antibody recently approved to treat relapsed/refractory B-cell acute lymphocytic leukemia, is the focus of a phase I trial in indolent lymphomas (such as FL and mantle cell lymphoma [MCL]). According to Dr. Zelenetz, overall response rates ranged from $70 \%$ to $80 \%$, and durable responses were seen with 4 to 8 weeks of blinatumomab monotherapy. In addition, blinatumomab has shown activity in DLBCL, "with a proportion of complete response rates in relapsed or refractory patients," he added.

Various antibody-drug conjugates are in development for B-cell lymphomas (Figure 2). Dr. Zelenetz took a closer look at 2 of them: the monomethyl auristatin $\mathrm{E}$ (MMAE) derivatives of anti-CD22 antibody (pinatuzumab vedotin) and the anti-CD79b antibody (polatuzumab vedotin). In the ROMULUS study, ${ }^{3}$ 122 patients with relapsed or refractory lymphoma $(\mathrm{FL}=41 ; \mathrm{DLBCL}=81)$ were randomized to receive rituximab plus the CD22 antibody drug conjugate or rituximab plus the CD79 antibody drug conjugate.

"The 2 drugs are very similar in their activity," explained Dr. Zelenetz. "There might be a little bit more activity in large cell lymphoma with the antiCD22 antibody and a little bit more activity with the anti-CD79b antibody in follicular lymphoma," he admitted, "but I would argue that picking the winner here is a matter of flipping a coin."

\section{Targeting Signaling Pathways and Apoptosis}

One of the more exciting signaling targets is the Bruton's tyrosine kinase (BTK). The BTK inhibitor ibrutinib has emerged as an effective treatment for several types of NHL, with approvals in chronic lymphocytic leukemia (CLL) and MCL and activity in DLBCL. The supporting data in MCL come from a phase II study in relapsed/refractory disease. ${ }^{4}$

"Even though responses are very rapid with this drug," explained Dr. Zelenetz, "the quality of response clearly improves with time." Benefits with ibrutinib were shown in both progression-free and overall survival. "Those patients who stay in remission tend to stay in remission for a long time," he added.

The combination of ibrutinib and rituximab is quite effective in patients with relapsed MCL. ${ }^{5}$ "The complete response rate is substantially higher than with single-agent ibrutinib," said Dr. Zelenetz. However, late toxicities with this regimen have been reported, including the development of atrial fibrillation and hypertension.

Ibrutinib is also active in relapsed or refractory DLBCL. A significant proportion of patients showed response and the responses were much more common among patients with $\mathrm{ABC}$-subtype compared to those with GBC-subtype, Dr. Zelenetz pointed out. Furthermore, the response to ibrutinib by mutational status suggested that its sensitivity does not require a BCR mutation and that it is not effective against a CARD11 mutation, noted Dr. Zelenetz. There were better responses to ibrutinib in those with a CD79 mutation than in those with wild-type CD79, he added.

Another therapeutic target is the PI3K pathway. The prototypical PI3K inhibitor idelalisib, approved for the treatment of CLL and small lymphocytic lymphoma (SLL), has shown activity in double-refractory (no response to rituximab and alkylating drugs) indolent NHL in a phase II study. ${ }^{6}$ The overall response rate in this study of 125 patients was $57 \%$, with $6 \%$ experiencing complete response. Idelalisib is relatively well tolerated over the long-term with diarrhea being the most common treatment-emergent adverse event, Dr. Zelenetz stated. Dr. Zelenetz posed a major question about these pathway inhibitors. "How do we integrate them earlier in the treatment in a way that fundamentally changes the natural history of the lymphomas we are treating?"

Turning to targeting apoptosis, Dr. Zelenetz highlighted the second-generation BCL-2 inhibitor venetoclax (ABT-199). Early studies with venetoclax have confirmed its activity and the durability of 


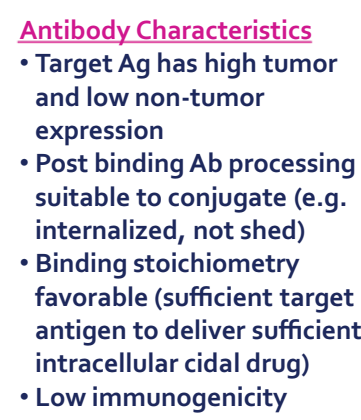

Antibody Characteristics

- Target Ag has high tumor and low non-tumor

Post binding $\mathrm{Ab}$ processing suitable to conjugate (e.g

internalized, not shed)

- Binding stoichiometry antigen to deliver sufficient

- Low immunogenicity

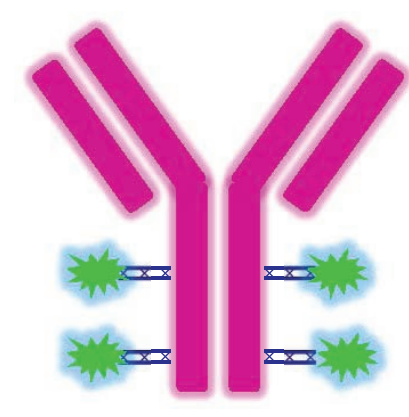

Linker Characteristics

- Stable in circulation and storage

- Readily cleavable in target cell

- Differential cleavage in tumor snf

non-tumor

- Drug conjugation to antibody does

not impair Ab binding
Conjugate Drug Characteristics

- Highly potent at low

concentrations

- Therapeutic margin is

significantly improved with

targeted delivery

- Free drug is minimal, although

potentially beneficial as

bystander effects kill

neighboring cells

- Soluble in aqueous solution

Figure 2 Ideal Characteristics of Immunoconjugates.

responses in patients with relapsed/refractory CLL, including those with unmutated immunoglobulin heavy chain variable region (IGHV) genes, del(17p), and fludarabine-refractory disease. ${ }^{7}$ Overall response rate was nearly $80 \%$ with a complete response rate of $23 \%$. Response rates were similar across the risk categories. "Progress-free survival is also promising with this agent and it is being evaluated in ongoing definitive studies in patients with CLL", Dr. Zelenetz pointed out.

Venetoclax also has shown activity in different subtypes of relapsed or refractory NHL. The overall response rates are variable and the most promising response rate was seen in patients with MCL. In the very early data, Dr. Zelenetz noted that about 70\% to $80 \%$ of patients with MCL had a response to this agent, and many ongoing studies are evaluating venetoclax in multiple NHL histologies.

\section{Immunomodulators and Checkpoint Inhibitors}

The immunomodulators have had their central place in the management of multiple myeloma but also have important activity in NHL. The immunomodulating agent lenalidomide in combination with rituximab has been evaluated in a phase II study in untreated indolent lymphomas. ${ }^{8}$ In the FL study population, $98 \%$ responded to the combination of lenalidomide and rituximab, with $87 \%$ achieving a complete response. Many patients in other subgroups responded as well (89\% marginal zone, 80\% SLL).
These data were confirmed in the CALGB 50803 study in patients with untreated FL. The ORR was $93 \%$ with $72 \%$ complete response rate, "which is a very impressive confirmation of a single-center study," declared Dr. Zelenetz.

In a study attempting to identify biomarkers predictive for therapeutic response, ${ }^{9}$ lenalidomide was evaluated in patients with relapsed or refractory DLBCL. The investigators found that the cell of origin had an impact on outcomes. A total of $52.9 \%$ of patients with a non-germinal center B-cell-like phenotype responded to lenalidomide compared with only $8.7 \%$ of patients with a germinal center B-cell-like phenotype. ${ }^{9}$ This suggests that the benefit of lenalidomide is restricted to non-germinal center DLBCL, added Dr. Zelenetz. The results of 2 subsequent studies confirmed that the addition of lenalidomide to $\mathrm{R}-\mathrm{CHOP}$ "appears to overcome the adverse impact of non-germinal center cell of origin in patients with DLBCL," he pointed out. The use of immune checkpoint inhibitors has a rationale in lymphoma due to the overexpression of a number of immune checkpoint proteins that are overexpressed on malignant cells and/or in the tumor microenvironment. Dr. Zelenetz briefly reviewed the preliminary data from clinical trials evaluating use of nivolumab (an anti PD-1 checkpoint inhibitor) in hematologic malignancies. In a study of nivolumab in 105 patients with relapsed or refractory hematologic malignancies, 29 of whom had B-cell lymphomas, the overall response rate was modest at $28 \%$, with a few complete responses and some patients 
Options for B-Cell Lymphomas

with stable disease. ${ }^{10}$ However, in the subgroups of patients with FL and DLBCL, "there is a hint that the activity is somewhat greater," he said. He added that these are very small numbers and larger confirmatory studies are needed to validate these findings. The most common side effects were fatigue, pneumonitis, and pruritus. "The safety profile [with nivolumab] appears to be much more favorable than [with] ipilimumab," said Dr. Zelenetz. "These drugs [immune check point inhibitors], because of their acceptable toxicity profile, are obviously good targets for combination with other treatments," Dr. Zelenetz concluded.

\section{References}

1. Czuczman MS, Hess G, Gadeberg OV, et al. Chemoimmunotherapy with ofatumumab in combination with CHOP in previously untreated follicular lymphoma. Br J Haematol 2012;157:438-445.

2. Sehn LH, Goy A, Offner FC, et al. Randomized phase II trial comparing GA101 (obinutuzumab) with rituximab in patients with relapsed CD20 indolent B-cell non-Hodgkin lymphoma: preliminary analysis of the GAUSS study [abstract]. Blood 2011;118:Abstract 269.

3. Morschhauser F, Flinn I, Advani RH, et al. Preliminary results of a phase II randomized study (ROMULUS) of polatuzumab vedotin or pinatuzumab vedotin plus rituximab in patients with relapsed/refractory non-Hodgkin lymphoma [abstract]. J Clin Oncol 2014;32:Abstract 8519.

4. Wang ML, Rule S, Martin P, et al. Targeting BTK with ibrutinib in relapsed or refractory mantle-cell lymphoma. N Engl J Med 2013;369:507-516.

5. Wang ML, Hagemeister F, Westin JR, et al. Ibrutinib and rituximab are an efficacious and safe combination in relapsed mantle cell lymphoma: preliminary results from a phase II clinical trial [abstract]. Blood 2014;124:Abstract 627

6. Gopal AK, Kahl BS, de Vos S, et al. PI 3 K $\delta$ inhibition by idelalisib in patients with relapsed indolent lymphoma. N Engl J Med 2014;370:1008-1018.

7. Seymour JF, Davids MS, Pagel JM, et al. ABT-199 (GDC-0199) in relapsed/ refractory chronic lymphocytic leukemia and small lymphocytic lymphoma: high response rates among patients with high risk disease features including unmutated IGHV [abstract]. Presented at the 2014 European Hematology Association Meeting; June 12-14, 2014; Milan, Italy.

8. Fowler NH, Davis RE, Rawal S, et al. Safety and activity of lenalidomide and rituximab in untreated indolent lymphoma: an open-label, phase 2 trial. Lancet Oncol 2014;15:1311-1318.

9. Hernandez-Ilizaliturri FJ, Deeb G, Zinzani PL, et al. Higher response to lenalidomide in relapsed-refractory diffuse large B-cell lymphoma in nongerminal center B-cell-like than in germinal center B-cell-like phenotype. Cancer 2011;117:5058-5066.

10. Lesokhin AM, Ansell SM, Armand P, et al. Preliminary results of a phase I study of nivolumab (BMS-936558) in patients with relapsed or refractory lymphoid malignancies [abstract]. Blood 2014;124:Abstract 291. 